\title{
Integração de imagens NOAA/AVHRR: Rede de cooperação para monitoramento nacional da safra de soja
}

\author{
Anibal Gusso ${ }^{1}$
}

\begin{abstract}
RESUMO
Uma avaliação inicial das condições do desenvolvimento da safra nacional, enquanto as plantas ainda estão nos campos, é altamente necessária para o cálculo correto das projeções na tomada de decisão e políticas relacionadas com o planejamento governamental e segurança alimentar. O objetivo deste trabalho foi avaliar a adequação dos dados NOAA/AVHRR (National Oceanic and Atmospheric Administration / Advanced Very High Resolution Radiometer) em detectar mudanças nas condições da vegetação, devidas à ocorrência de estresse hídrico, na soja, por meio de uma combinação do índice NDVI (Normalized Difference Vegetation Index) e da LST (Land Surface Temperature). Os dados LST e NDVI foram combinados e comparados pixel a pixel, sobre uma área de cultivo de soja, no Rio Grande do Sul. A relação teórica inversa prevista na combinação de LST e NDVI foi detectada. Foi observado que ocorre um aumento médio na LST em uma safra de ciclo normal (de 301,02 K para 308,36 K), quando comparada a uma safra sob condição de estresse hídrico, no desenvolvimento da cultura. Uma redução média do NDVI foi observada no ciclo normal (de 0,65 para 0,53 ), comparada com uma safra sob efeitos ocasionados pela estiagem no desenvolvimento da cultura. Foi observado maior correlação da produtividade municipal com LST $(\mathrm{R} 2=0,78)$ do que com o NDVI $(\mathrm{R} 2=0,59)$. Os resultados obtidos indicam que a integração de imagens do sensor AVHRR, proveniente de diferentes instituições, proporciona a adequada combinação espacial e temporal dos dados LST e NDVI, a fim de detectar a ocorrência de estresse hídrico, bem como sua intensidade, caracterizando as condições do ciclo de desenvolvimento da soja.
\end{abstract}

Palavras-chave: sensoriamento remoto, estiagem, temperatura da superfície terrestre, índice de vegetação.

\section{ABSTRACT}

\section{Integration of NOAA/AVHRR images: Cooperation network towards national soybean crop monitoring}

An early assessment of national crop development conditions while the plants are still in the fields is highly needed to calculate correctly projections for decision-making and policies related to government planning and food security. The aim of this study was to evaluate the suitability of NOAA/AVHRR (National Oceanic and Atmospheric Administration / Advanced Very High Resolution Radiometer) to detect changes in vegetation conditions, due to water stress during soybean crop, by means a combination of Land Surface Temperature (LST) and Normalized Difference Vegetation Index (NDVI). Both LST and NDVI data were combined and compared in a pixel basis over a soybean crop area in Rio Grande do Sul State. The predicted theoretical inverse relationship for the combination of LST and NDVI was detected. An average increase of LST was observed in a normal crop cycle ( from $301.02 \mathrm{~K}$ to $308.36 \mathrm{~K}$ ) compared to a crop cycle under water stress condition. An average reduction in NDVI was observed for normal crop cycle development (from 0.65 to 0.53 ) compared to a crop cycle under drought-induced effects. It was observed a higher correlation of municipality yield with LST $(\mathrm{R} 2=0.78)$ than NDVI $(\mathrm{R} 2=0.59)$. Results obtained indicate that the aggregation of AVHRR images, from

Recebido para publicação em 31/05/2011 e aprovado em 14/02/2013.

Físico, Mestre. Centro de Ciências do Sistema Terrestre - Instituto Nacional de Pesquisas Espaciais (INPE), Avenida dos Astronautas, 1758, Jardim da Granja, Caixa Postal 515, 12227-010, São José dos Campos, São Paulo, Brasil. anibal.gusso@inpe.br 
different institutions, provides the appropriate combination of spatial and temporal data LST and NDVI in order to detect the occurrence of drought stress, as well as its intensity, characterizing the conditions of the crop cycle development of soybean.

Key words: remote sensing, drought, land surface temperature, vegetation index.

\section{INTRODUÇÃO}

Em escala global, o gerenciamento do setor agrícola tem-se tornado cada vez mais sofisticado, exigindo informações continuamente atualizadas, de maneira a se obter levantamentos confiáveis sobre o desempenho das safras, antecipadamente ao período da colheita, estratégias de aprimoramento das políticas públicas de abastecimento e de equilíbrio entre oferta e demanda (Figueiredo, 2005; Gusso et al., 2012).

Em um panorama de mudanças climáticas, parâmetros agrometeorológicos da superfície terrestre devem sofrer alterações nas próximas décadas (Siqueira et al, 2000; Pinto, 2009; Batistti \& Naylor, 2009; Peterson et al., 2012). Períodos prolongados de baixa ocorrência de precipitação ou exposição a temperaturas elevadas produzem desequilíbrio nos sistemas ecológico e hidrológico e têm impactos severos na produção agrícola (Kogan et al., 2003; Karnieli et al., 2010; Board \& Kahlon, 2011), com efeitos em vários níveis da organização social.

No Brasil, aumentos na frequência de eventos extremos, como a ocorrência de altas temperaturas e a redução na precipitação, deverão produzir efeitos severos na produtividade agrícola (Streck \& Alberto, 2006; Lobell et al., 2008), especialmente de soja e milho. Como decorrência disso, as distorções e indefinições nas políticas agrícolas devem amplificar as perdas e estabelecer barreiras na formação de metodologias para o financiamento agrícola e políticas assistenciais adequadas.

No Estado do Rio Grande do Sul (RS), a grande variabilidade interanual da precipitação, verificada em função, principalmente, dos fenômenos El Niño e La Niña, é o principal fator responsável pelas oscilações de produtividade na região sul (Buriol et al., 1977, Fontana et al., 2002, Melo et al., 2004, Ferreira, 2006), sendo que as culturas mais afetadas geralmente são as da soja e do milho (Fontana et al., 2002) na safra de verão.

O efeito prolongado da estiagem na safra da soja em 2004/2005, quando comparada com a safra de 2002/2003, apresentou uma redução de $76 \%$ na produção de grãos (IBGE, 2010). Mundialmente, a ocorrência de estiagens deverá ocasionar perdas severas na produção agrícola, com potenciais impactos na economia (UNEP, 2009). As- sim, é de grande importância a implementação de um sistema nacional de monitoramento contínuo dos cultivos, para uma avaliação mais detalhada da vulnerabilidade e sustentabilidade dos processos adaptativos às novas condições e das futuras demandas internacionais por commodities agrícolas. Para tanto, são imprescindíveis estratégias de gerenciamento para redução dos impactos e melhor aproveitamento das condições climáticas favoráveis (Ferreira, 2006). Entretanto, é importante salientar que embora o aumento na frequência de estiagens e seus efeitos tenha sido o maior interesse nos últimos anos, os potenciais impactos de perdas no setor agrícola, decorrentes de sensíveis aumentos nas temperaturas sazonais, vêm ganhando interesse (Batistti \& Naylor, 2009).

O sensoriamento remoto proporciona informações sistemáticas e de alta qualidade espacial e temporal sobre a superfície terrestre (Liu \& Kogan, 2002). Na última década, índices espectrais de vegetação, particularmente derivados dos sensores AVHRR (Advanced Very High Resolution Radiometer), a bordo dos satélites da série NOAA (National Oceanic and Atmospheric Administration) têm demonstrado grande potencial no monitoramento do ciclo de desenvolvimento agrícola (Liu and Kogan, 2002; Kogan et al., 2003; Esquerdo et al., 2011). Estes dados têm sido especialmente utilizados em levantamentos do NDVI (Normalized Difference Vegetation Index) e da LST (Land Surface Temperature), para compor estudos de séries históricas (Karnieli et al., 2010). Uma ampla gama de variáveis físicas e climáticas afeta o NDVI, a LST e a relação entre cobertura vegetada e temperatura (Karnieli et al., 2010). Segundo Nemani \& Running, (1997), diferenças na LST também têm aplicações na observação da taxa de crescimento das plantas. Sandholt et al. (2002) salientam que o uso do índice de vegetação e da temperatura da superfície aborda uma relação entre o vigor vegetativo e a resposta termal característica do dossel.

A estruturação de uma rede de cooperação institucional proporciona vantagens, ao gerar mapas em áreas de menor densidade de cobertura por dados agrometeorológicos, para todo o país. No Brasil, como exemplo, estão em funcionamento contínuo antenas de Brasília (Instituto Nacional de Meteorologia-INMET), Campinas (Universidade de Campinas-UNICAMP) e Porto Alegre (Universi- 
dade Federal do Rio Grande do Sul-UFRGS) dentre outras instituições (Figura 1). Gusso et al., (2007) e Esquerdo et al., (2011) testaram a obtenção dos dados NOAA/AVHRR para estimativa do NDVI e da LST e observaram que estes sensores são adequados para o mapeamento da LST em tempo hábil à tomada de decisão, diferentemente dos dados meteorológicos pontuais, obtidos em abrigos meteorológicos ou por estações automáticas, que, frequentemente, estão incompletos ou não disponíveis, para uma avaliação em tempo hábil (Kogan, 2002; Sims et al., 2008).

Este estudo apresenta uma proposta de integração de dados de satélite, por meio de uma rede de cooperação institucional, para monitoramento da produção brasileira de soja, com base no índice de vegetação e no comportamento térmico da superfície, obtidos do NOAA/AVHRR. O objetivo foi caracterizar a relação que se estabelece na combinação desses dois parâmetros físicos, em associação, como agentes agregadores das condições predominantes de desenvolvimento da vegetação, durante as safras que apresentaram maior variabilidade da produtividade no RS.

\section{MATERIAL E MÉTODOS}

\section{Área do estudo experimental no Rio Grande do Sul}

A área de estudo experimental é o Estado do RS, um dos maiores produtores e exportadores de grãos do Brasil. Nesse Estado, a área de mais intensa produção agrícola está na região norte, com aproximadamente $76700 \mathrm{~km}^{2}$ (Figura 2), e abrange 223 municípios (Gusso et al., 2012). O RS é dominado pelos climas subtropical e temperado, respectivamente caracterizados pelos tipos $C f a$ e $C f b$ de Köppen (1948). O mês mais quente é janeiro com temperatura média de $23,7^{\circ} \mathrm{C}$ e a média de precipitação pluvial ao longo do ano é de 1.680 mm, sendo que o mês que apresenta maiores médias de precipitação pluviométrica é outubro, com 173 mm (Melo, 2003). A época de semeadura das culturas do milho e da soja no sul do Brasil começa em setembro e vai até novembro. Dependendo da época da semeadura, entre setembro e novembro, a vegetação atinge seu máximo do final de janeiro até início de março (Gusso et al., 2012), quando alcança os seus valores máximos do NDVI (Fontana et al., 2002).

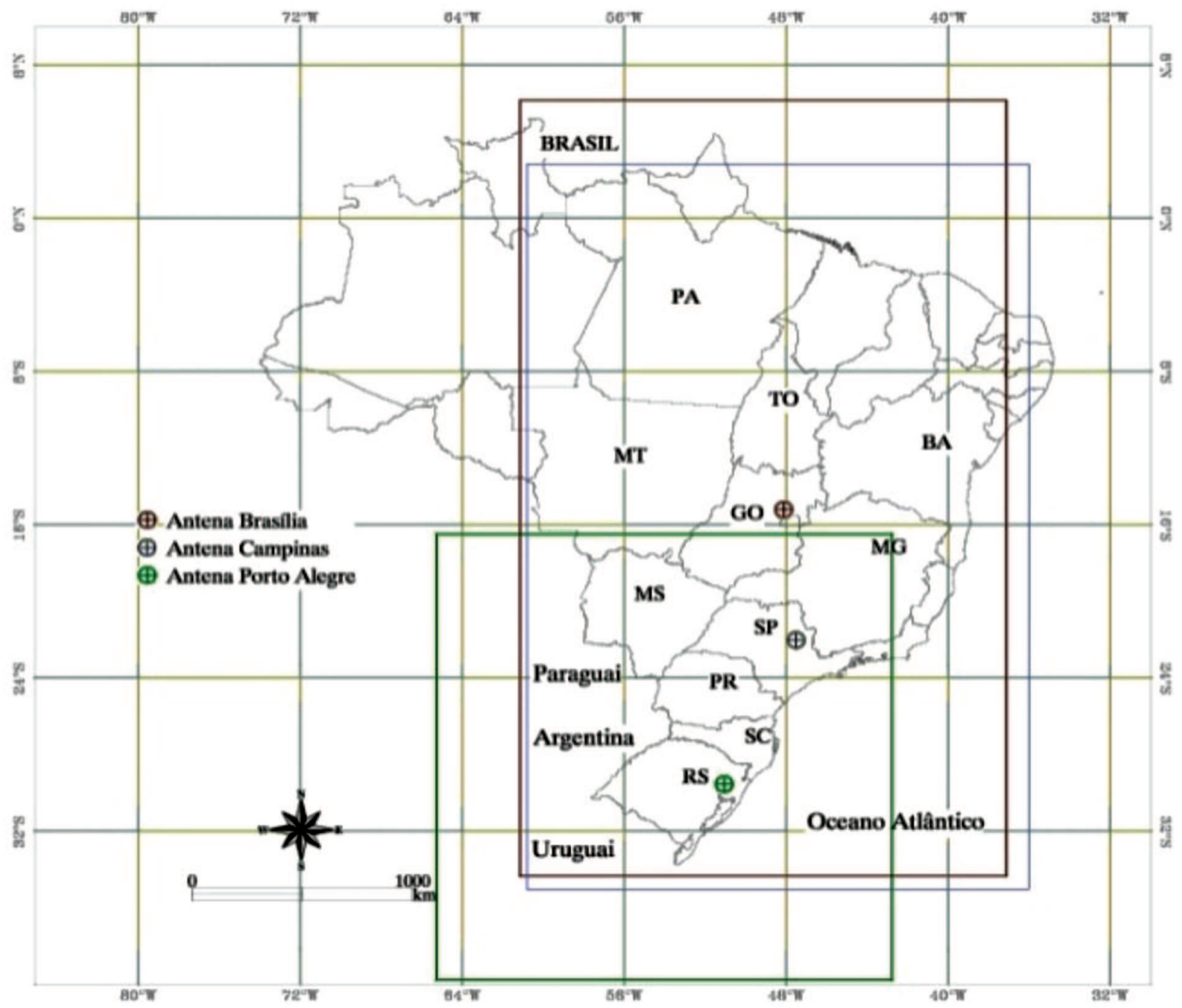

Figura 1. Área aproximada de cobertura e a posição das antenas de recepção de dados NOAA/AVHRR sobre o território brasileiro. 
Considerando o período da floração, enchimento de grãos e maturação físiológica, que ocorre nos meses de janeiro e fevereiro para a maioria dos cultivares, foi escolhido o mês de fevereiro de cada safra, na análise da combinação do máximo NDVI e máxima LST. Com base nas composições mensais de máximo NDVI e máxima LST, obtidos na região de grande produção, foram considerados os período da safra de 2002/2003, que correspondeu a uma elevada produtividade média estadual $(2800 \mathrm{Kg} /$ ha); uma safra com médio efeito de estiagem em 2003/ 2004, com produtividade reduzida (1400 Kg/ha); e uma safra sob severo efeito da estiagem em 2004/2005, que resultou em uma produtividade média estadual muito baixa (600 Kg/ha), segundo o IBGE (2010). Foram extraídos os valores, pixel a pixel, do NDVI e LST, considerando a área de intensa produção agrícola que responde por quase $70 \%$ da produção da soja no Estado, a qual foi delimitada pelo arquivo vetorial da malha municipal oficial do IBGE, conforme Figura 2.

\section{Dados de pré-processamento}

O processamento das imagens NOAA/AVHRR e a posterior integração e quantificação dos dados multitemporais em NDVI e LST foi obtido de imagens captadas pela estação de recepção de dados NOAA, instalada no Campus do Vale da UFRGS (Universidade Federal do Rio Grande do Sul).

Para a geração de dados do NDVI, a partir dos dados NOAA/AVHRR, foi utilizado o satélite NOAA-16, com passagem em horário pouco após o meio-dia local, e por proporcionar, simultaneamente, a obtenção dos dados da LST proximamente ao horário de máxima temperatura do dia. Imagens brutas foram submetidas aos procedimentos de calibração de pós-lançamento para a obtenção dos dados de NDVI. Esse procedimento consiste na calibração dos dados dos instrumentos, nas bandas 1 e 2, por causa da degradação, pelo tempo de operação, dos sensores, e da exposição às condições severas do espaço, como, também, da geometria de imageamento da superfície terres-



Figura 2. Área de estudo e a posição das estações meteorológicas utilizadas dentro da área de intenso cultivo da soja. 
tre. Esses procedimentos, descritos por Rao \& Chen (1999), estão de acordo com as recomendações do NESDIS (National Environmental Satellite, Data and Information Service).

O NDVI é calculado pela diferença entre as reflectâncias detectadas nas bandas do visível $(0,58$ à $0,68 \mu \mathrm{m})$ e infravermelho próximo $(0,725$ a $1,1 \mu \mathrm{m})$, dividida pelas somas dessas quantidades, como definido por Rouse et al. (1974). Desse cálculo, em pixel, resultam valores que variam entre -1.0 e +1.0 (Chen et al., 2002) e tem ele sido amplamente utilizado como medida na avaliação de parâmetros sobre as condições de desenvolvimento da vegetação. Em condições normais de desenvolvimento, a vegetação vigorosa absorve a radiação no visível para realizar a fotossíntese e a reflete, no infravermelho-próximo, por causa do espalhamento da luz pelos tecidos mesofílicos e pelo conteúdo de água interno da folha. Com base nessas propriedades, a diferença entre o infravermelho-próximo e o visível torna-se grande (Kogan, 2002). A partir das imagens individuais diárias do satélite NOAA-16, foram geradas composições de imagens de NDVI, obtidas pelo cálculo do MVC (Maximum Value Composite) de Holben (1986). O mesmo método foi aplicado na obtenção das composições da máxima LST. Para obtenção dos dados da LST, foram utilizados os procedimentos de transformação dos dados digitais em radiância, por meio da equação de Planck, descritos em Sullivan (1999) e Gusso et al. (2007).

A LST pode ser determinada a partir da detecção da radiação de ondas longas, emitida na faixa do infravermelho, que deixa a superfície terrestre e é detectada pelo

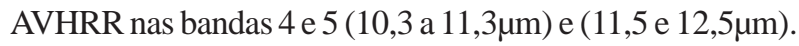
Medidas da temperatura, obtidas por satélites, estão relacionados com a temperatura cinética da superfície irradiadora sendo esta considerada, para efeito dos cálculos iniciais, como um corpo-negro ideal (Sobrino et al., 2001, Gusso et al., 2007). Foram utilizados dados obtidos da temperatura de brilho da banda 4, do NOAA/ AVHRR, conforme os estudos de Nemani \& Running, (1997), Kogan, (2001), Kogan, (2002) e Kogan et al., (2003), que aprimoraram técnicas que relacionam o NDVI e a LST no estudo do desenvolvimento da vegetação agrícola.

A precipitação pluvial e a temperatura determinam os padrões de evapotranspiração real e balanço hídrico (Board \& Kahlon, 2011).

O parâmetro LST é chave nos processos físicos terrestres em escala regional e global (Wan et al., 2004). Considerando-se que a atividade fisiológica está mais diretamente relacionada com a temperatura da folha, do que com a temperatura do ar circundante, a LST é a componente física da temperatura mais adequada ( $\operatorname{Sims} e t$ al., 2008).

\section{Combinação do NDVI e LST}

Alguns trabalhos já exploraram uma relação entre índices de vegetação e o comportamento térmico da superfície, obtido por dados de sensoriamento remoto (Nemani et al., 1993; Goetz, 1997; Gupta et al., 1997; Lambin \& Ehrlich, 1995; Sandholt et al., 2002; Sims et al., 2008). Alguns desses estudos têm sido desenvolvidos sobre firmes bases teóricas (Moran et al., 1994), outros, como também no presente trabalho, estão fortemente fundamentados em parametrizações empíricas de imagens de satélite obtidas por sensores colocados em órbita (Sandholt $e t$ al., 2002; Kogan, 2002; Wan et al., 2004), mas exigem grande quantidade de dados para assegurar que as condições de contorno estejam adequadamente estabelecidas.

No RS, diversos trabalhos têm demonstrado a alta correlação entre o déficit hídrico e as condições meteorológicas cumulativas, durante o período de crescimento e de desenvolvimento das culturas com a produção agrícola no RS (Fontana et al., 2001; Fontana $e t$ al., 2002). Liu \& Kogan (2002) observam que é uma condição física que estabelece a relação matemática inversa entre o NDVI e LST e que essa relação impõe que as mais altas LST, obtidas no período de máximo desenvolvimento, estão associadas às condições mais desfavoráveis de crescimento da vegetação. Nesse sentido, Liu \& Kogan (2002) também observam que, de maneira simétrica, os maiores valores do NDVI, representativos do maior vigor vegetativo, devem estar associados às LST mais baixas, indicando melhores condições de crescimento da vegetação. Entretanto, o NDVI não é, preferencialmente, um indicador cumulativo do estresse hídrico, pois a vegetação pode permanecer verde após o início da condição de estresse hídrico (Sandholt et al., 2002; Wan et al., 2004). Goetz (1997) também observou que decorre um atraso (lag-time) entre a diminuição da disponibilidade hídrica e a resposta dos valores do NDVI, mas ocorre uma resposta mais imediata com o aumento da temperatura do dossel (Nemani \& Running, 1997; Goetz, 1997), sendo indiretamente, um dos primeiros sinais de redução na disponibilidade hídrica.

No caso da soja, a redução das chuvas durante essas fases fenológicas exerce influência direta na produtividade final (Ferreira, 2006). Mesmo quando o NDVI indica uma característica típica da vegetação verde, uma sensível redução na disponibilidade hídrica promove o fechamento dos estômatos, o que ocasiona uma redução na capacidade das plantas de reterem a energia refletida por meio de absorção, afetando também a capacidade da realização da fotossíntese. Como resposta ao desequilíbrio no balanço do fluxo de energia, provocado pela baixa circulação de água na estrutura interna (mesofílica) da vegetação, a temperatura do dossel aumenta rapidamente (Nemani \& Runing, 1997; Wan et al., 2004). Já na situação 
oposta, em que predominam superfícies bem úmidas, a relação entre NDVI e LST está mais diretamente relacionada com a umidade da superfície, pelo aumento da inércia térmica do solo (Friedl \& Davis, 1994).

Em períodos secos, um suprimento insuficiente de água no interior da planta leva ao sobreaquecimento da vegetação, o que intensifica o impacto da deficiência hídrica (Liu \& Kogan, 2002) pela elevação da temperatura (Allen et al., 2010). Isto ocorre por que as condições biofísicas da superfície terrestre determinam as temperaturas do solo e da vegetação, assim como a temperatura do ar próxima à superfície do solo (Ouaidrari et al., 2002). Na Amazônia, Brando et al. (2010) analisaram os efeitos de uma severa estiagem, em 2005, e observaram que a exposição à radiação solar incidente induziu o fechamento dos estômatos, mesmo da vegetação nativa. Entretanto, aqueles autores não utilizaram dados de LST, o que não permitiu localizar temporalmente os efeitos do aumento da temperatura no dossel.

Kogan (2001), analisando a relação entre índices de vegetação e a LST, observou que o período da floração da vegetação agrícola sazonal, é mais sensível à temperatura do que ao estresse hídrico. O período de florescimento (estádios R1 ao final do R5) e enchimento de grãos (estádios R5 ao final do R7) são os mais sensíveis às flutuações das condições físicas na superfície terrestre (Kogan et al., 2003; Ferreira, 2006; Board \& Kahlon, 2011), o que, no $\mathrm{RS}$, normalmente ocorre nos meses de janeiro a março (Ferreira, 2006).

Em sistemas agrícolas tão homogêneos como em campos de cultivo, a radiação incidente afeta a resistência dos estômatos para transpiração, alterando a relação entre calor sensível e calor latente (Nemani \& Running, 1997; Sandholt et al., 2002). O estresse provocado pela ocorrência de altas temperaturas tem ação sobre a atividade fotossintética (Carmo-Silva et al., 2012). Temperaturas acima de $32^{\circ} \mathrm{C}$ reduzem a taxa de crescimento da planta, em especial as plantas do tipo C3, como a soja, o feijão e o café, resultando em menor produtividade (Pinto, 2009; Board \& Kahlon, 2011). Temperaturas ótimas para soja, entre 26 e 36 graus centígrados (Pinto, 2009; Board \& Kahlon, 2011), são as mais frequentemente citadas.

\section{RESULTADOS E DISCUSSÃO}

As Figuras 3, 4 e 5 apresentam dados da precipitação mensal acumulada de outubro a março, em relação à normal climatológica, comparados com os dados de LST e NDVI do mês de fevereiro, nas respectivas safras. Os dados de precipitação acumulada mostram as diferentes condições hídricas entre as safras analisadas. Na safra 2002/ 2003, o período de novembro de 2002 a fevereiro de 2003 caracterizou-se por precipitações acima da média da região. Em contraste, a precipitação acumulada, na mesma época, nas duas safras seguintes, ficou abaixo da média.
Os valores mais baixos de NDVI, tipicamente entre zero e 0,2 , não foram relevantes neste experimento, pois representavam corpos d'água, solo úmido ou sem cobertura vegetal, grandes áreas urbanas ou mesmo contaminação residual de nuvens. Fontana et al., (2002) geraram estatísticas a partir do mesmo tipo de dados NDVI sobre áreas agrícolas, nessa região, e observaram que durante este período as áreas analisadas exibem valor médio de 0,55 , com desvio padrão de 0,023 . Portanto, muito baixa probabilidade (menor que $1 \%$ ) de ocorrência de valores menores que 0,2.

A Figura 3 apresenta a distribuição de 2002/2003 como representativa das melhores condições de desenvolvimento de uma safra. A partir da Tabela 1, observam-se mais de $95 \%$ dos valores do NDVI acima de 0,5 e mais de 95\% valores da LST abaixo de $305 \mathrm{~K}$. Analisando-se a distribuição dos pontos nas Figuras 4 e 5, em relação aos da Figura 3, é possível observar que há uma migração intensa de pontos para a direita, com a maior parte reposicionando-se entre 295 e $315 \mathrm{~K}$. E um maior desvio padrão da LST (1,67 na Figura 3; 3,44 na Figura 4 e 3,16 na Figura 5), configurando um efeito de espalhamento, caracterizando assim, a distribuição também observada nos trabalhos de Sandholt et al., (2002). Na Figura 5, o efeito de espalhamento é intensificado. Ocorre um deslocamento generalizado dos pontos, com reagrupamento em direção às maiores LST, para a borda da região seca, assumindo a configuração do ressecamento da vegetação e elevação da LST (Sandholt et al., 2002), por causa das condições prolongadas do estresse hídrico, como prevê a relação física teórica estabelecida a priori. As Figuras 3, 4 e 5 também exibem pequenas parcelas de pontos espalhados com NDVI abaixo de 0,4 e com LST em torno dos $300 \mathrm{~K}$, nas safras 2002/2003 e 2003/2004, que correspondem a áreas úmidas e corpos d’água rasos. Na safra 2004/2005, pode ser observado um espalhamento desses pontos para maiores LST, indicando o aquecimento de toda a superfície da área estudada.

Na safra 2002/2003, LST maiores que 305 K correspondem a apenas $1 \%$ das ocorrências, conforme se pode depreender da Tabela 1. Nos meses de setembro a março de 2005, predominou uma condição de precipitação pluvial abaixo da média, sendo que, no acumulado de dezembro a março de 2005, observa-se que todas as estações meteorológicas estão sob influência de estiagem severa, com o posicionamento de mais de $85 \%$ dos 71000 pontos acima de $305 \mathrm{~K}$. Entretanto, em ambas as safras sob efeito de estiagem, observa-se um grupo de pontos com NDVI acima de 0,5, mesmo tendo LST acima dos $305 \mathrm{~K}$. Este resultado, na safra 2004/2005, sugere que, para algumas parcelas da área estudada a redução do índice de vegetação não foi seguida na proporção prevista de aumento da LST, possivelmente, por causa da predominância do efei- 
to de sobreaquecimento generalizado da superfície vegetada por uma onda de calor e não diretamente relacionada com a forçante imposta pelas condições prolongadas de deficiência hídrica.

Nemani \& Running, (1997) observam que as condições físicas de dissipação da energia entre calor latente e calor sensível são os principais mecanismos associados à LST. Isso pode indicar que a perda de produtividade, por ocorrência de estiagem, pode estar associada ao efeito acoplado de duas forçantes distintas nas safras analisadas, ou seja, uma redução inicial na disponibilidade hídrica, seguida de um aumento da temperatura, agravada
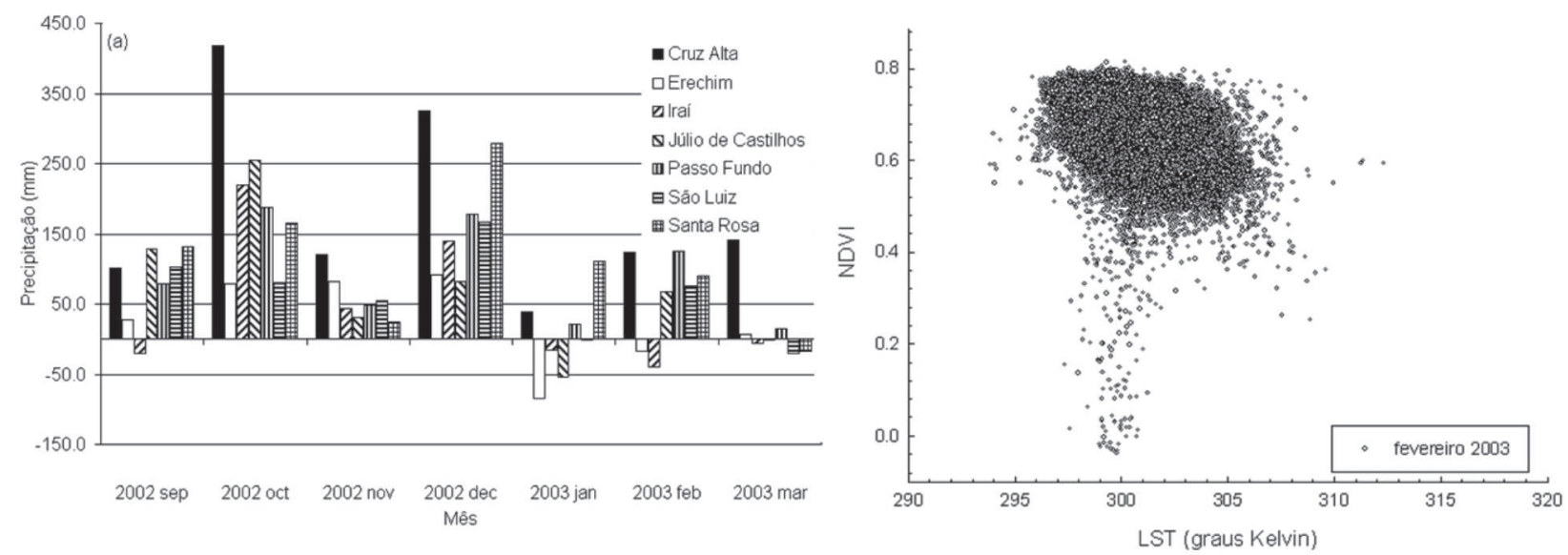

Figura 3. Diferença entre a precipitação mensal acumulada e a Normal Climatológica com o Diagrama de dispersão entre os valores de LST e NDVI, no mês de fevereiro, nas melhores condições de desenvolvimento (2002/2003).
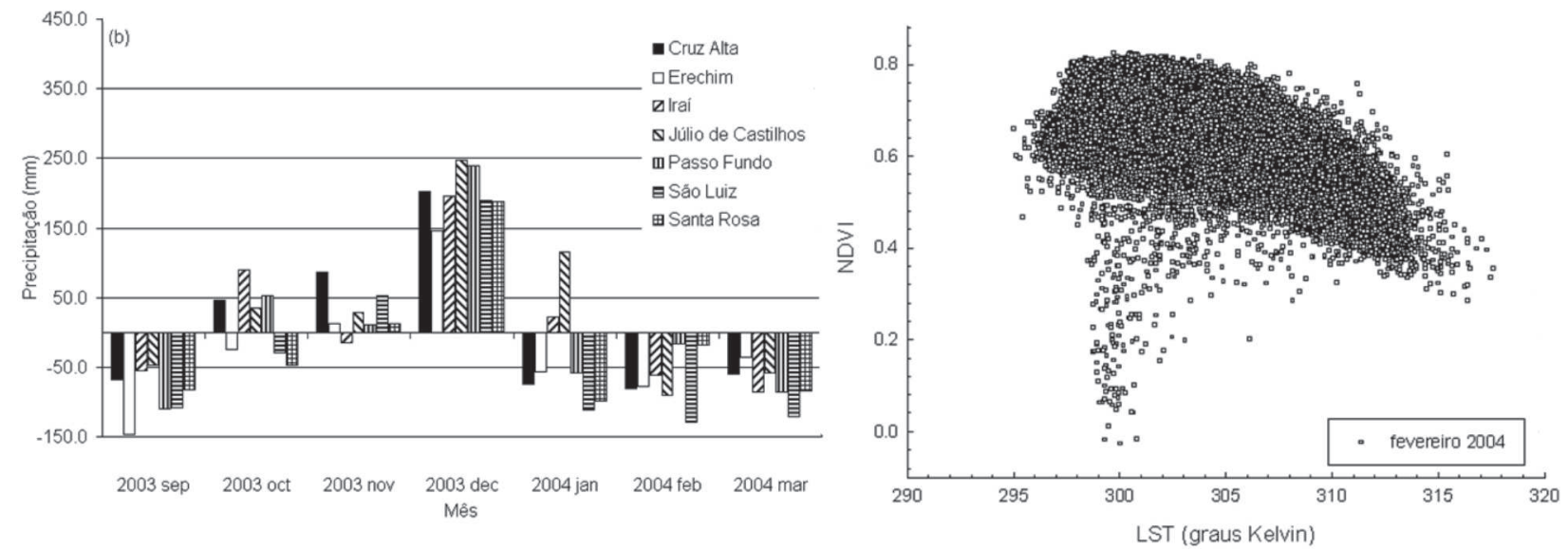

Figura 4. Diferença entre a precipitação mensal acumulada e a Normal Climatológica com o Diagrama de dispersão entre os valores de LST e NDVI, no mês de fevereiro, em condições de médio efeito de estiagem (2003/2004).
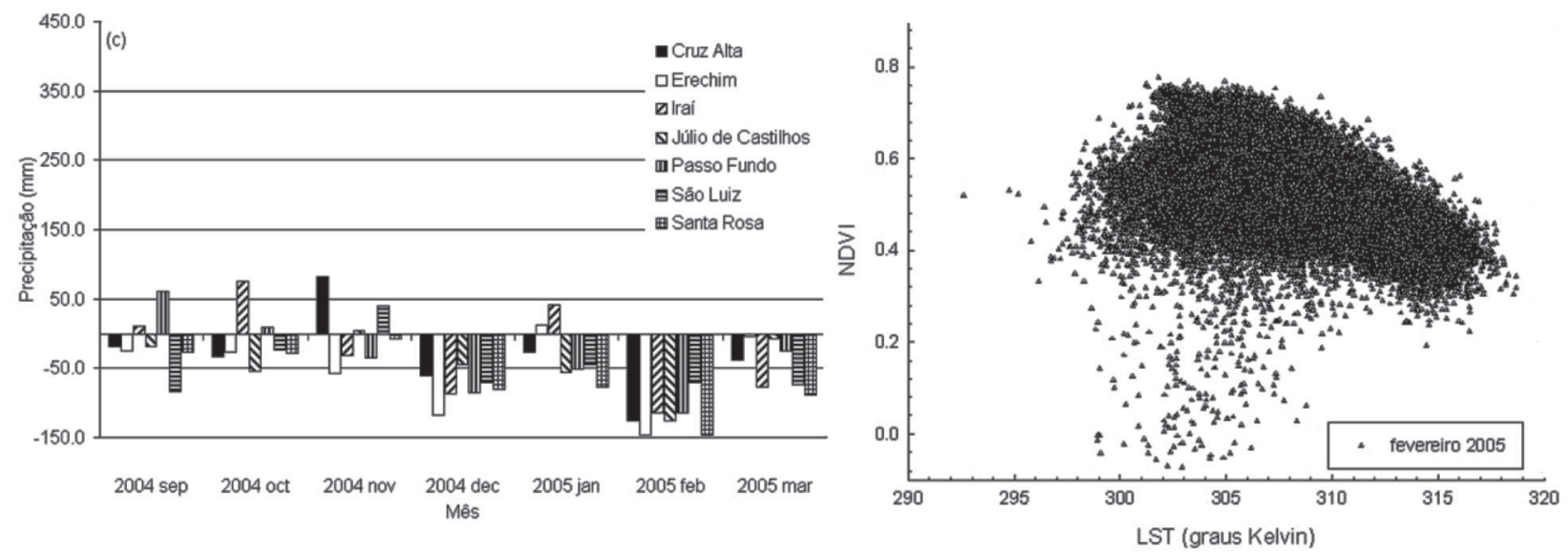

Figura 5. Diferença entre a precipitação mensal acumulada e a Normal Climatológica com o Diagrama de dispersão entre os valores de LST e NDVI, no mês de fevereiro, em condições de severo efeito de estiagem (2004/2005).

Rev. Ceres, Viçosa, v. 60, n.2, p. 194-204, mar/abr, 2013 
pelas condições de troca de energia, em função, principalmente, do vento, da umidade do ar e da exposição à radiação incidente (Nemani \& Running, 1997; Wan et al., 2004; Peterson et al., 2012). Nessa situação, a implementação de estratégias e políticas públicas de planejamento, quanto a época de semeadura e à utilização de técnicas de irrigação, como apontado em Melo et al., (2004), são extremamente importantes, considerando-se que ondas de calor possam ter intensidade potencializadas pela ocorrência de estiagem inibindo a atividade fotossintética (Carmo-Silva et al., 2012).

$\mathrm{Na}$ análise da estatística geral, na Tabela 1, vê-se que, com a grande quantidade de pontos analisados em cada safra (71000 pixels), mesmo com pequenas variações dos valores médios da LST e do NDVI, de uma safra em relação à outra, essas variações devem ser consideradas relevantes. O Teste-t heterocedástico não indicou probabilidade significativa de semelhança entre os conjuntos de dados do NDVI ou LST, nas safras estudadas. Uma diferença importante no NDVI só é observada na comparação de fevereiro de 2003 com fevereiro de 2005, em que a média do NDVI $(0,65)$ cai para 0,53 . Já com relação à LST, o aumento da dispersão dos pontos, aumento nos valores médios e nos máximos, proporcionalmente à severidade da estiagem, fica bem evidenciado. Apesar disso, os valores médios e máximos do NDVI, de fevereiro de 2003 em relação a fevereiro de 2004, são praticamente iguais. Possivelmente, como um efeito positivo do sensível aumento da radiação incidente em fevereiro de 2004 sobre cultivos irrigados. Entretanto, uma análise mais detalhada é necessária. A Tabela 1 mostra que as duas safras sob efeito de estiagem, mesmo com intensidades diferentes, apresentam semelhança na dispersão dos valores de NDVI. Fica evidente, no entanto, como o aumento no desvio padrão da LST (Tabela 1), parametrizado em um período específico, é útil como indicador de um processo desfavorável de desenvolvimento da vegetação agrícola.

A Figura 6 mostra dados agregados das três safras analisadas, em uma relação da produtividade municipal da soja obtida do IBGE e comparada com a LST média municipal $\left(\mathrm{R}^{2}=0,78\right)$ e, a Figura $6 \mathrm{~b}$, ao NDVI médio municipal $\left(\mathrm{R}^{2}=0,59\right)$. Fica evidente que os maiores valores da LST (predominantemente da safra 2004/2005), estão as- sociados às mais baixas produtividades, enquanto os menores valores do NDVI estão associados às menores produtividades. Os dados do NOAA/AVHRR evidenciam, nas Figuras 6a e 6b, que as médias municipais de temperaturas, acima de aproximadamente $306 \mathrm{~K}$ e NDVI menores que 0,5 , estão associadas às mais baixas produtividades (menores que $1000 \mathrm{Kg} / \mathrm{ha}$ ), caracterizando uma condição específica no desenvolvimento da vegetação da cultura da soja no mês de fevereiro, com impactos na produção.

\section{Rede de cooperação institucional: área de aplicação proposta}

Dados diários, obtidos de antenas posicionadas sobre o território brasileiro, formadas por uma rede de cooperação institucional, se forem conjugados, podem fornecer informações valiosas, na tomada de decisão em tempo hábil, para aplicações agrícolas de quase todo o território agrícola nacional. A grande área abrange as Latitudes com coordenadas: $06^{\circ} 00^{\prime} \mathrm{S}$ e $39^{\circ} 00^{\prime} \mathrm{S}$ e as Longitudes: $65^{\circ} 00^{\prime}$ $\mathrm{W}, 36^{\circ} 00^{\prime} \mathrm{W}$ (Figura 1). Outros estados de menor expressão agrícola e territórios estrangeiros de intensa produção de soja, como as regiões de Córdoba e Santa Fé, na Argentina, o Uruguai e o Paraguai, também poderiam ser monitorados. Aárea proposta de monitoramento nacional continuado abrange todos os Estados de maior expressão agrícola, como Bahia, Goiás, Mato Grosso, Mato Grosso do Sul, Minas Gerais, Paraná, Rio Grande do Sul, Santa Catarina e São Paulo.

Os mapas de variabilidade do NDVI e da LST (Figuras 7a e 7b) mostram a ampla área de aplicação proposta sobre o território brasileiro, na vigência de uma rede de cooperação institucional. Esta pode ser obtida como resultado da agregação de dados de imagens do NOAA/AVHRR. Salienta-se, na Figura 7b, a região centro norte do Estado do MT, GO e DF. Os valores moderados da LST, em novembro, são representativos de condições favoráveis de desenvolvimento da vegetação. No norte do PR, é possível observar focos de LST mais elevadas que são representativas do período de pré-plantio. A Figura $7 \mathrm{~b}$ também mostra as amplitudes extremas da LST, em função das condições da vegetação e de cobertura do solo, notadamente em Estados do nordeste que não são evidenciadas quando analisados apenas os dados de ima-

Tabela 1. Estatística básica (média, desvio padrão, valor mínimo e máximo) da distribuição da nuvem de pontos nas safras analisadas entre LST (Land Surface Temperature) e NDVI (Normalized Difference Vegetation Index)

\begin{tabular}{|c|c|c|c|c|c|c|}
\hline & \multicolumn{2}{|c|}{ Fevereiro 2003} & \multicolumn{2}{|c|}{ Fevereiro 2004} & \multicolumn{2}{|c|}{ Fevereiro 2005} \\
\hline & LST (K) & NDVI & LST (K) & NDVI & LST (K) & NDVI \\
\hline Média & 301,02 & 0,65 & 303,68 & 0,66 & 308,36 & 0,53 \\
\hline Desv. Padrão & 1,67 & 0,06 & 3,44 & 0,08 & 3,16 & 0,08 \\
\hline Mínimo & 293,83 & $-0,03$ & 295,01 & $-0,02$ & 292,60 & $-0,07$ \\
\hline Máximo & 312,30 & 0,81 & 317,59 & 0,82 & 318,68 & 0,77 \\
\hline
\end{tabular}



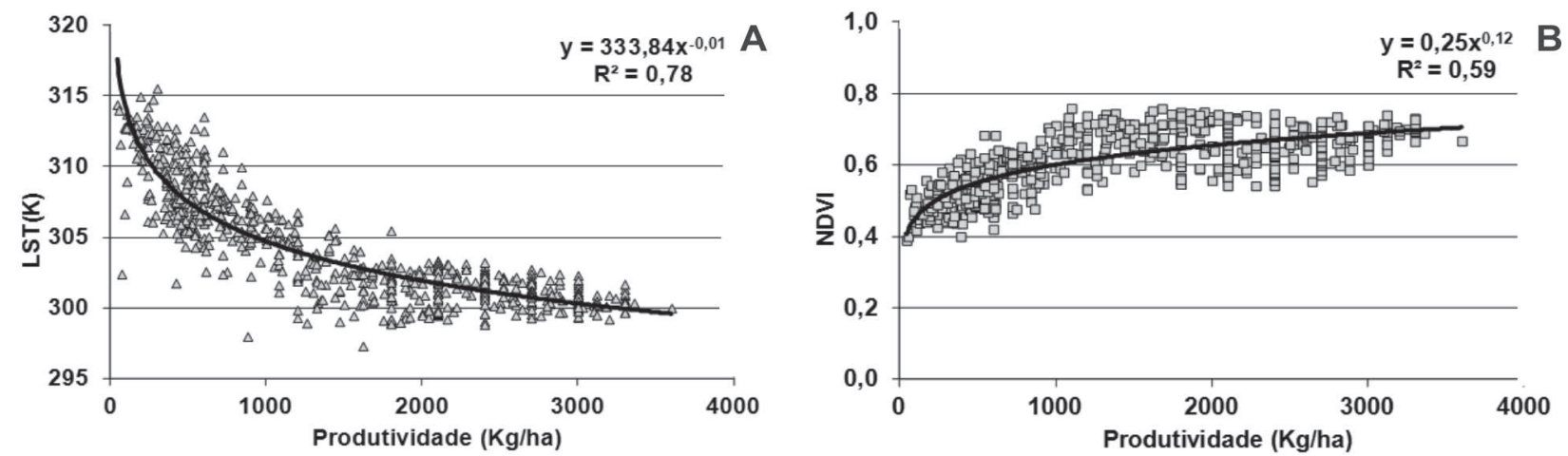

Figura 6a. Dados agregados das safras 2002/2003, 2003/2004 e 2004/2005, mostrando a variação da média municipal da LST em relação à produtividade municipal, obtida do IBGE. Figura 6b. Dados agregados das safras 2002/2003, 2003/2004 e 2004/2005, mostrando a variação da média municipal do NDVI em relação à produtividade municipal, obtida do IBGE.
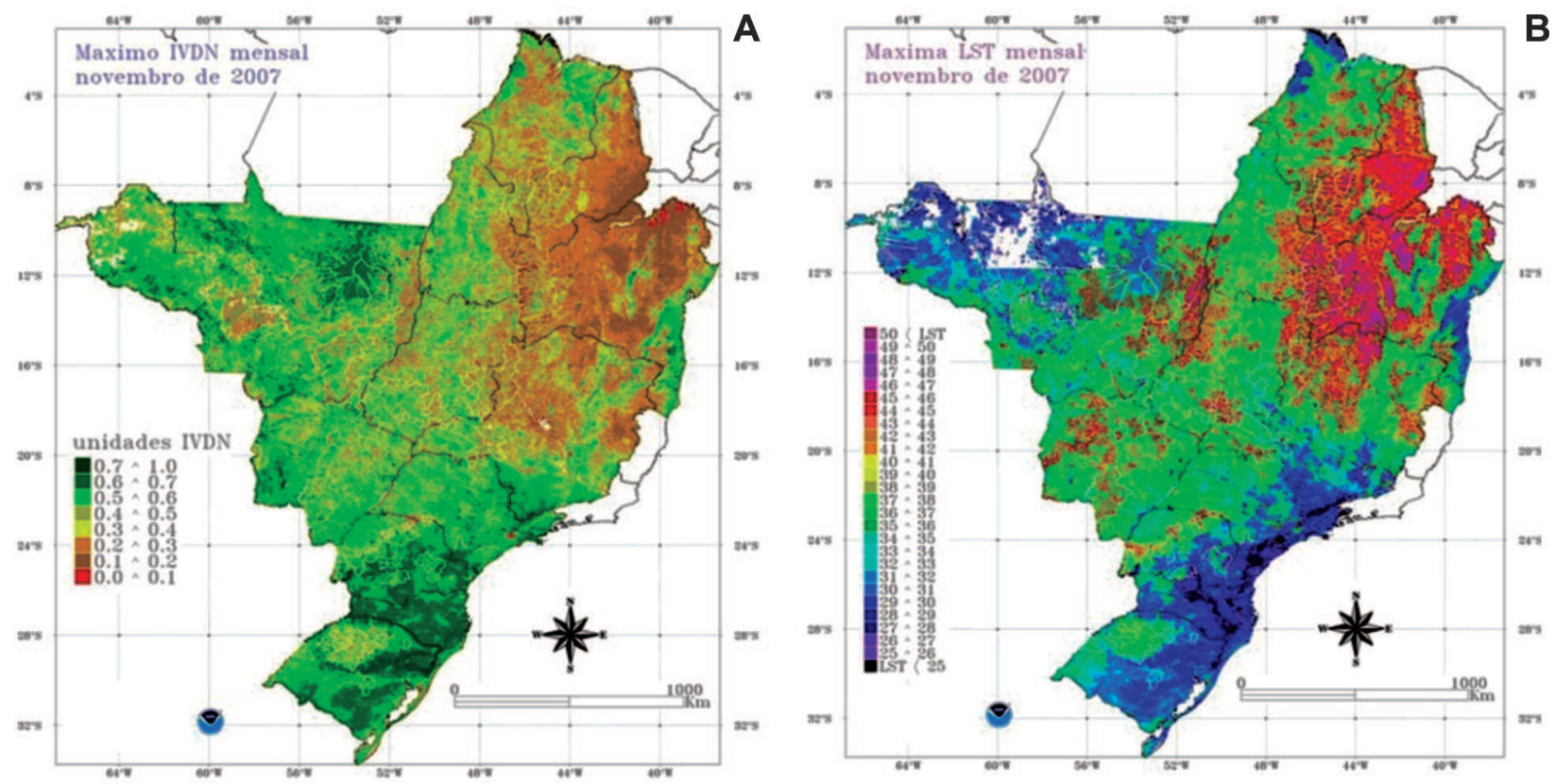

Figura 7a. Composição de máximo valor do NDVI mensal (novembro de 2007), obtidos pela agregação de imagens do INMET/ Brasília e UFRGS/Porto Alegre. Figura 7b. Composição de máxima LST mensal (novembro de 2007), obtida com a agregação de imagens do INMET/Brasília e UFRGS/Porto Alegre.

gens de índices de vegetação. Esses aspectos térmicos da superfície terrestre e que caracterizam condições adversas para atividade rural, como as observadas na região nordeste, podem assim ser analisados mais adequadamente pelos tomadores de decisão com vistas ao desenvolvimento de políticas públicas mais eficazes.

\section{CONCLUSÕES}

A relação matemática inversa que se evidencia, neste trabalho, entre o NDVI e a LST associada à produtividade, confirma a expectativa teórica.

Sensores NOAA/AVHRR têm grande potencial na caracterização espacial e temporal das condições intermediárias de desenvolvimento das safras agrícolas.
A sensibilidade introduzida pela variável LST $\left(R^{2}=0,78\right)$, analisada neste estudo, revela ser um indicador útil dos diferentes níveis das condições da vegetação, quando em processo desfavorável de desenvolvimento, sugerindo que esta é uma abordagem robusta e promissora. Assim, esta abordagem pode ser explorada para outras regiões produtoras do país, principalmente como indicador espacial de queda na produtividade. Entretanto, é de grande importância realizar estudos adicionais que contemplem o acompanhamento do processo de estresse hídrico, associado, ou não à ocorrência de ondas de calor a partir do início da safra, de maneira a permitir avaliação mais detalhada da vulnerabilidade frente a esse componente físico e seus impactos na sustentabilidade da produção agrícola. 


\section{AGRADECIMENTOS}

À Fundação Estadual de Pesquisa Agropecuária do Rio Grande do Sul (FEPAGRO), à Universidade Federal do Rio Grande do Sul (UFRGS), ao Instituto Nacional de Meteorologia (INMET) e à Universidade de Campinas (UNICAMP). Em especial aos Editores e revisores anônimos pelas valiosas contribuições.

\section{REFERÊNCIAS}

Allen CD, Macalady AK, Chenchouni H, Bachelet D, McDowell N, Vennetier M, Kitzberger T, Rigling A, Breshears DD, Hogg EH, Gonzalez P, Fensham R, Zhangm Z, Castro J, Demidova N, Lim J-H, Allard G, Running SW, Semerci A \& Cobb N (2010) A global overview of drought and heat-induced tree mortality reveals emerging climate change risks for forests. Forest Ecology and Management, 259:660-684.

Board JE \& Kahlon CS (2011) Soybean yield formation: what controls it and how it can be improved. In: El-Shemy, H.A. (Ed.) Soybean Physiology and Biochemistry, InTech Open Access Publisher, Rijeka, Croatia, 1-36.

Batistti DS \& Naylor RL, (2009) Historical warnings of future food insecurity with unprecedented seasonal heat. Science, $323: 240-244$

Brando PM, Goetz SJ, Baccini A, Nepstad DC, Beck PSA \& Christman MC (2010) Seasonal and interanual variability of climate and vegetation indicies across the Amazon. Proceedings of National Academy of Science of USA, 107:14685-14690.

Buriol GA, Estefanel V \& Ferreira M (1977) Cartas mensais e anual das chuvas do Estado do Rio Grande do Sul. Revista do Centro de Ciências Rurais, 7:55 82.

Carmo-Silva AE, Gore MA, Andrade-Sanchez P, French AN, Hunsaker DJ \& Salvucci ME (2012) Decreased $\mathrm{CO}_{2}$ availability and inactivation of Rubisco limit photosynthesis in cotton plants under heat and drought stress in the field. Environmental and Experimental Botany, 83:1-11.

Chen PY, Srinivasan R, Fedosejevs G \& Narasimhan B (2002) An automated cloud detection method for daily NOAA-14 AVHRR data for Texas, USA. International Journal of Remote Sensing, 23:2939-2950.

Esquerdo JCDM, Zullo J, \& Antunes, JFG (2011) Use of NDVI/ AVHRR time-series profiles for soybean crop monitoring in Brazil. International Journal of Remote Sensing, 32:3711-3727.

Figueiredo DC (2005) Projeto GeoSafras - aperfeiçoamento do sistema de previsão de safras da CONAB. Revista de Política Agrícola, 14:110-120.

Fontana DC, Weber E, Ducati JR, Berlato MA, Guasselli LA \& Gusso A (2002) Monitoramento da cultura da soja no centro-sul do Brasil durante La Niña de 1998/2000. Revista Brasileira de Agrometeorologia, 10:343-351.

Fontana DC, Berlato MA, Lauschner MH \& Melo RW (2001) Modelo de estimativa de rendimento de soja no Estado do Rio Grande do Sul. Pesquisa Agropecuária Brasileira, 36:399-403.

Friedl MA \& Davis FW (1994) Sources of variation in radiometric surface temperature over a tallgrass prairie. Remote Sensing of Environment, 48:1-17.

Goetz SJ (1997) Multi-sensor analysis of NDVI, surface temperature and biophysical variables at a mixed grassland. International Journal of Remote Sensing, 18:71-94.
Gupta RK, Prasad S, Sesha-Sai MVR \& Viswanadham TS (1997) The estimation of surface temperature over an agricultural area in the state of Haryana and Panjab, India, and its relationship with the Normalized Difference Vegetation Index (NDVI), using NOAA-AVHRR data. International Journal of Remote Sensing, 18:3729-3741.

Gusso A, Formaggio AR, Rizzi R, Adami M \& Rudorff BFT (2012) Method for soybean area estimation based on multi-temporal MODIS/EVI images. Pesquisa Agropecuária Brasileira, 47:425435 .

Gusso A, Fontana DC \& Gonçalves GA (2007) Mapeamento da temperatura da superfície terrestre com uso do sensor NOAA/ AVHRR. Pesquisa Agropecuária Brasileira, 42:231-237.

Holben BN (1986) Monitoring characteristics of maximum-value composite images from temporal AVHRR data. International Journal of Remote Sensing, 7:1417-1434.

IBGE (2010). Sistema IBGE de Recuperação Automática. Disponível em: <http://www.sidra.ibge.gov.br/>. Acessado em: 05 de setembro de 2010.

Karnieli A, Nurit A, Pinker TR, Anderson M, Imhoff ML, Gutman GG, Panov N \& Goldberg A (2010) Use of NDVI and Land Surface Temperature for Drought Assessment: Merits and Limitations. Journal of Climate, 23:618-633.

Kogan FN (2001) Operational space technology for global vegetation assessment. Bulletin of American Meteorological Society, 82:1949-1964

Kogan FN (2002) World droughts in the millennium from AVHRRbased vegetation health indices. Eos, Transactions, American Geophysical Union, 83:557-564.

Kogan FN, Gitelson A, Zakarin E, Spivak L \& Lebed L (2003) AVHRR-Based Spectral Vegetation Index for Quantitative Assessment of Vegetation State and Productivity: Calibration and Validation. Photogrammetric Engineering \& Remote Sensing, 69:899-906

Köppen W (1948) Climatologia: con un Estúdio de los Climas de la Tierra. Fondo de Cultura Econômica. México. 466p

Lambin EF \& Ehrlich D (1995) Combining vegetation indices and surface temperature for land-cover mapping at broad spatial scales. International Journal of Remote Sensing, 16:573-579.

Lobell DB, Burke MB, Tebaldi C, Mastrandrea MD, Falcon WP \& Naylor RL (2008) Prioritizing climate change adaptation needs for food security in 2030. Science, 319:607-609.

Liu WT \& Kogan F (2002) Monitoring Brazilian soybean production using NOAA/AVHRR based vegetation indices. International Journal of Remote Sensing, 23:1161-1180.

Melo RW (2003) Desenvolvimento de um modelo agrometeorológico-espectral de estimativa do rendimento da soja para o Estado do Rio Grande do Sul. Dissertação de Mestrado. Universidade Federal do Rio Grande do Sul, Porto Alegre. 130p.

Melo RW, Fontana DC \& Berlato MA (2004) Indicadores de produção de soja no Rio Grande do Sul comparados ao zoneamento agrícola. Pesquisa Agropecuária Brasileira, 39:1167-1175.

Moran MS, Clarke TR, Inoue Y \& Vidal A (1994) Estimating crop water deficit using the relation between surface-air temperature and spectral vegetation index. Remote Sensing of Environment, 49:246-263

Nemani R \& Running S (1997) Land cover characterization using multi-temporal Red, Near-IR and thermal-IR data from NOAA/ AVHRR. Ecological Applications, 7:79-90.

Nemani R, Pierce L, Running S \& Goward S (1993) Developing satellite-derived estimates of surface moisture status. Journal of Applied Meteorology, 32:548-557. 
Ouaidrari H, Goward SN, Czajkowski KP, Sobrino JA \& Vermote E (2002) Land surface temperature estimation from AVHRR thermal infrared measurements: An Assessment for the AVHRR Land Pathfinder II Data Set. Remote Sensing of Environment, 81:114-128.

Pinto HS (2009) Adaptation in the brazilian agricultural sector. In: Marengo, JA (Ed.) Climate change, extreme weather and climate events in Brazil. Lloyd's Group Report. p. 35-51.

Peterson TC, Stott PA \& Herring S (2012) Explaining extreme events of 2011 from a climate perspective. Bulletin of the American Meteorological Society, 93:1041-1067.

Rao CRN \& Chen J (1999) Revised post-launch calibration of the visible and near-infrared channels of the Advanced Very High Resolution Radiometer on board the NOAA-14 spacecraft. International Journal of Remote Sensing, 20:3485-3491.

Rouse JW, Haas RH, Schell JA, Deering DW \& Harlan JC (1974) Monitoring the vernal advancement and retrogradation (greenwave effect of natural vegetation), Report. Disponível em: 〈http://archive.org/details/nasa_techdoc_19740022555> Acessado em: 27 de março de 2011.

Sandholt L, Rasmunssen K, Andersen J (2002) A simple interpretation of the surface temperature/vegetation index space for assessment of surface moisture status. Remote Sensing of Environment, 79:213-224.
Sims DA, Rahman AF, Cordova VD, El-Masri BZ, Baldocchi DD, Bolstad PV, Flanagan LB, Goldstein AH, Hollinger DY, Misson L, Monson RK, Oechel WC, Schmid HP, Wofsy SC \& Xu L (2008) A new model of gross primary productivity for North American ecosystems based solely on the enhanced vegetation index and land surface temperature from MODIS. Remote Sensing of Environment, 112:1633-1646.

Siqueira OJW, Steinmetz S, Ferreira MF, Costa AC, Wozniak MA (2000) Mudanças climáticas projetadas através dos modelos GISS e reflexos na produção agrícola brasileira. Revista Brasileira de Agrometeorologia, 8:311-320.

Sobrino JA, Raissouni N \& Li Z-L (2001) A comparative study of land surface emissivity retrieval from NOAA data. Remote Sensing of Environment, 75:256-266.

Streck NA \& Alberto CM (2006) Estudo numérico do impacto da mudança climática sobre o rendimento de trigo, soja e milho. Pesquisa Agropecuária Brasileira, 41:1351-1359.

Sullivan JT (1999) New radiance-based method for AVHRR thermal channel nonlinearity corrections. Journal of Remote Sensing, 20:3493-3502.

UNEP (2009). The Environmental Food Crisis. Disponível em: <http://www.grida.no/publications/rr/food-crisis/> Acessado em: 20 de novembro de 2011.

Wan Z, Wang P \& Li X (2004) Using MODIS Land Surface Temperature and Normalized Difference Vegetation Index products for monitoring drought in the southern Great Plains, USA. International Journal of Remote Sensing, 25:61-72. 